\title{
Chitosan Oligosaccharide Induces Resistance to Pseudomonas syringae pv. tomato DC3000 in Arabidopsis thaliana by Activating Both Salicylic Acid- and Jasmonic Acid-Mediated Pathways
}

\author{
Xiaochen Jia, ${ }^{1}$ Haihong Zeng, ${ }^{1,2}$ Wenxia Wang, ${ }^{1}$ Fuyun Zhang, ${ }^{2}$ and Heng Yin ${ }^{1,+}$ \\ ${ }^{1}$ Liaoning Provincial Key Laboratory of Carbohydrates, Dalian Institute of Chemical Physics, Chinese Academy of Sciences, \\ Dalian 116023, China; and ${ }^{2}$ College of Food Science and Engineering, Dalian Ocean University, Dalian 116023, China
}

Accepted 1 June 2018.

\begin{abstract}
Chitosan oligosaccharide (COS) is an effective plant immunity elicitor; however, its induction mechanism in plants is complex and needs further investigation. In this study, the ArabidopsisPseudomonas syringae pv. tomato DC3000 (hereafter called DC3000) interaction was used to investigate the induction effect and the underlying mechanisms of COS. COS is effective in inducing resistance to DC3000 in Arabidopsis, and our results demonstrate that treatment with COS 3 days before DC3000 inoculation provided the most effective resistance. Disease severity in jarl (jasmonic acid [JA]-deficient mutant), NahG, and sid2 (salicylic acid [SA]-deficient mutants) suggest both the SA and JA pathways are required for the Arabidopsis response to DC3000. COS pretreatment induced resistance in wild type (WT), jar1, and also, although to a lesser degree, in NahG and sid2 plants, implying that the SA and JA pathways play redundant roles in COS-induced resistance to DC3000. In COS-pretreated plants, expression of genes related to the SA pathway (PR1, PR2, and $P R 5)$ and SA content increased in both WT and jar1. Moreover, expression of genes related to the JA pathway (PDF1.2 and VSP2) and JA content both increased in WT and NahG. In conclusion, COS induces resistance to DC3000 in Arabidopsis by activating both SA- and JA-mediated pathways, although SA and JA pathways play redundant roles in this COS-induced resistance.
\end{abstract}

The interaction of Arabidopsis thaliana and Pseudomonas syringae pv. tomato DC3000 (hereafter called DC3000) has been used for many years as a typical plant pathosystem to study plant defense response (Jovanovic et al. 2017; Katagiri et al. 2002; Liu et al. 2015; Naseem et al. 2014). DC3000 is a hemibiotrophic bacterium that was initially isolated from tomato (Cuppels 1986) and, thus, is not a natural pathogen of Arabidopsis. However, under laboratory conditions with a high bacterial titer inoculation, DC3000 invades Arabidopsis leaf tissue, multiplies rapidly in intercellular spaces, and causes

${ }^{\dagger}$ Corresponding author: Heng Yin; E-mail: yinheng@dicp.ac.cn

Funding: This work was supported by National Key R\&D Program of China (2017YFD0200900), National Natural Science Foundation of China (31370811), H. Yin was supported by CAS Youth Innovation Promotion Association (2015144) and Special Fund of Dalian city for Distinguished Young Scholars (2015R010).

○ 2018 The American Phytopathological Society visible disease symptoms on infected leaves (Katagiri et al. 2002; Velásquez et al. 2017).

When pathogens attack plants, various signaling pathways are activated, including the salicylic acid (SA)- and the jasmonic acid/ethylene (JA/ET)-mediated signaling pathways (Kouzai et al. 2018; Zhang et al. 2017). In both basal and induced resistance in plants, the SA and JA/ET pathways influence each other by acting in synergistic and antagonistic ways (Betsuyaku et al. 2018; Koornneef and Pieterse 2008; Zhang et al. 2015). According to previous studies, plants that overaccumulate SA or have been treated with exogenous SA are more resistant to DC3000, suggesting that SA plays an essential role in Arabidopsis resistance to DC3000 (Todesco et al. 2010; Velásquez et al. 2017; Wilson et al. 2017). Generally, SA and JA pathways work in an antagonistic way; however, some studies have found that both SAand JA-mediated pathways are required for Arabidopsis resistance to DC3000. During DC3000 infection, both the SA and JA pathway-related genes in Arabidopsis are upregulated, leading to SA and JA accumulation in infected leaves (Betsuyaku et al. 2018; Hossain et al. 2008; Khare et al. 2016; Kloek et al. 2001).

In plant-pathogen interactions, microbe-associated molecular patterns (MAMPs) such as chitin and flagellin are perceived by plant receptors that activate plant innate immunity to defend against pathogen invasion (Poncini et al. 2017). Chitosan, composed of $\beta$-1,4-glucosamines, is one of the most abundant polysaccharides in the world. Chitosan oligosaccharide (COS) is a product of chitosan hydrolysis and is an effective MAMP that induces plant innate immunity (Yin et al. 2010, 2016). According to previous studies, COS serves as a potent immunity elicitor in many plants, including tobacco (Zhang et al. 2012), camellia (Li and Zhu 2013), wheat (Wang et al. 2015), oilseed rape (Yin et al. 2013), tomato (Zhang and Chen 2009), soybean (Valdés-López et al. 2014), Arabidopsis (Jia et al. 2016), and rice (Yang et al. 2017).

Chitosan's induction mechanism on fungi is revealed by inserting itself into the minor groove of DNA and activating DNA damage responses to potentiate plant immunity (Hadwiger 2015; Hadwiger and Tanaka 2017). This signal perception mode is quite different from chitin oligomers, which are recognized by receptors at the plasma membrane by affecting transcription factors without directly acting on DNA (Desaki et al. 2018; Hadwiger and Tanaka 2017; Squeglia et al. 2017). Recently, a work has revealed that fluorescence-labeled COS are enriched on the wheat leaf cell surface, and several candidates that have the potential to be COS receptors were found (Liu et al. 2018). Thus, besides acting on DNA directly, $\mathrm{COS}$ receptors on plant plasma membrane maybe also exist. 
Although the COS signal perception mechanism is by DNA damage responses or receptor recognition, the downstream signal transduction pathways are similar. As a broad-spectrum plant immunity elicitor, COS activates different signaling pathways for defense against pathogen invasion. Previous studies have demonstrated that COS treatment enhances the expression of JArelated genes in Brassica napus (Yin et al. 2006) and increases JA content in tomato (Doares et al. 1995), rice (Rakwal et al. 2002), and rapeseed (Yin et al. 2013). These results reveal that COS enhances plant immunity mainly through the JA-mediated pathway. However, when COS-treated Arabidopsis or tobacco plants are infected with the Tobacco mosaic virus (TMV), only the SAmediated pathway is activated (Jia et al. 2016; Yafei et al. 2009). Similar results were observed in COS-pretreated rice, where infection with the Southern rice black-streaked dwarf virus (SRBSDV) dramatically increased PR1 transcription level (Yang et al. 2017). Thus, because of the complexity of the COS-induced immune signaling network, the topic requires further research.

In this study, Arabidopsis-P. syringae pv. tomato DC3000 interaction was used to further reveal the complex mechanisms of COS-induced immunity. The involvement of SA and JAmediated pathway in COS-induced resistance to DC3000 in Arabidopsis were investigated by using SA- or JA-deficient mutants, including jarl (JA pathway-deficient mutant), NahG, and sid2 (SA pathway-deficient mutants).

\section{RESULTS}

COS induces resistance to DC3000 in Arabidopsis.

As a broad-spectrum plant immunity regulator, COS is effective in inducing resistance against many viral or bacterial infections in plants. According to previous studies, $50 \mathrm{mg}$ of COS per liter is the most effective concentration for pretreatment to induce immunity against many plant pathogens in many plants, including tobacco-TMV, Arabidopsis-TMV, oilseed rape-S. sclerotiorum, and rice-SRBSDV (Jia et al. 2016; Li and Zhu 2013; Yang et al. 2017; Yin et al. 2008; Zhao et al. 2007). However, the optimal COS pretreatment time differs for different plant-pathogen interactions: 1 day for tobacco-TMV and Arabidopsis-TMV interactions and 3 days for rapeseed-S. sclerotiorum interaction (Jia et al. 2016; Yin et al. 2008; Zhao et al. 2007). Thus, we investigated the optimal timing for pretreatment with $\operatorname{COS}(50 \mathrm{mg} / \mathrm{liter})$ in Arabidopsis in order to induce immunity against DC3000.

COS was sprayed onto Arabidopsis plants 1,2, and 3 days before and $0,1,2$, and 3 days after DC 3000 inoculation. The disease phenotypes were recorded at 4 days after DC3000 inoculation. Based on the disease index and bacterial growth data, Arabidopsis plants treated with COS prior to DC3000 inoculation displayed reduced disease symptoms compared with those treated with COS after DC3000 inoculation. The optimal treatment time was observed at 3 days prior to DC3000 inoculation (Fig. 1A and B).

The expression of DC3000 marker genes ( $a v r P t o B$ and recA) were measured to evaluate the infection status of DC3000 in infected leaves. avrPtoB is a DC3000 effector that enters the host cell to interfere with the kinase domains of plant pattern recognition receptor complexes, resulting in enhanced pathogen growth and enhanced DC3000 virulence (Abramovitch and Martin 2005; Chien et al. 2013; Kraus et al. 2016). recA is typically used as an internal reference gene when measuring relative expression of the genes in DC3000; thus, it can be used to quantify DC3000 accumulation in infected plant tissue (Scalschi et al. 2014). The
A

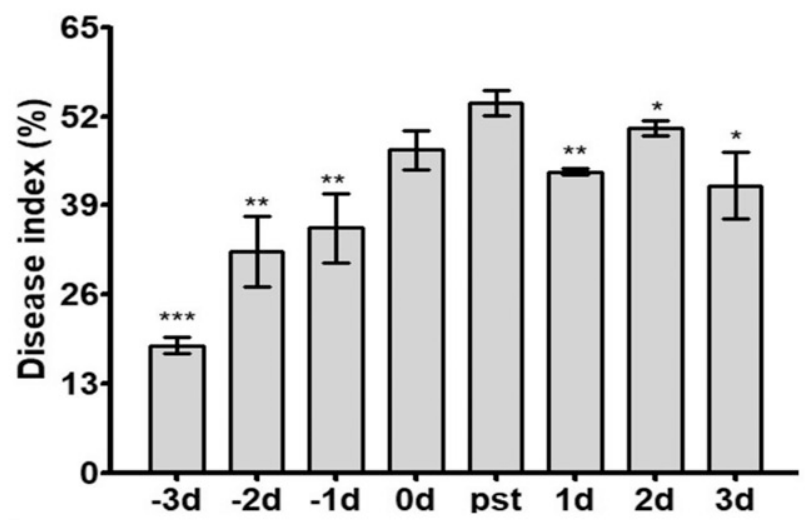

c

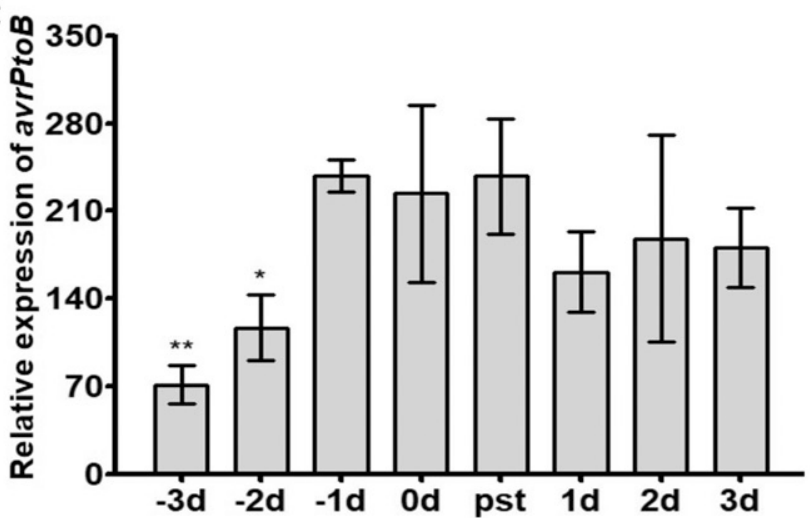

B

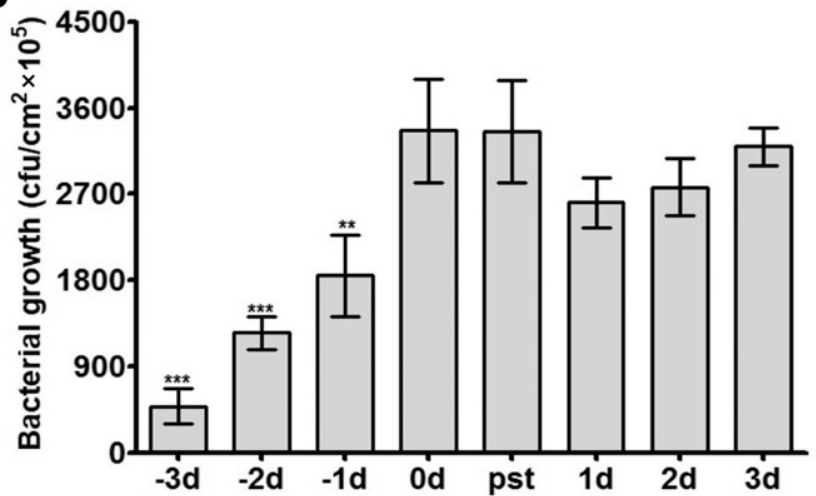

D

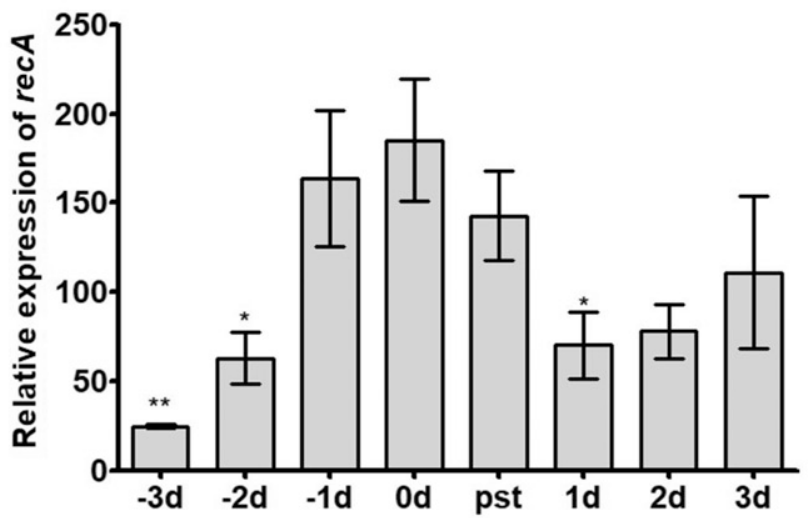

Fig. 1. The optimal treatment time for chitosan oligosaccharide (COS) in inducing resistance to Pseudomonas syringae pv. tomato DC3000 in Arabidopsis. COS was sprayed onto Arabidopsis plants for each of the 3 days before (-3d, -2d, -1d) and 3 days after (0d, 1d, 2d, 3d) DC3000 inoculation. Plants inoculated with DC3000 without COS treatment were used as control (pst). A, Disease index. B, Bacterial population per square centimeter of leaf tissues. C, Expression of the DC3000 marker genes $a v r P t o B$ and D, recA were detected. Values are the means \pm standard deviation in three independent measurements. One asterisk (*) indicates significant difference at $P<0.05$, two (**) at $P<0.01$, and three (***) at $P<0.001$. 
expression of $a v r P t o B$ and $r e c A$ were consistent with the disease index and bacterial population, further confirming that pretreatment of plants with a $50 \mathrm{mg} /$ liter concentration of COS 3 days before DC3000 inoculation leads to better disease control (Fig. 1C, D). Furthermore, these indexes from different levels were highly matched, so they are all usable for the symptoms calculation index in the DC3000 infection of Arabidopsis.

\section{SA or JA deficiency impaired resistance to DC3000} in Arabidopsis.

We utilized the immune signaling pathway-deficient mutants jarl, NahG, and sid2 to investigate the roles of SA and JA pathways in DC3000 infection of Arabidopsis.
Four days after DC3000 inoculation, typical disease symptoms included extensive chlorosis and multiple necrotic lesions appeared in inoculated leaves. The observed phenotypes (Fig. 2A) and disease index (Fig. 2B) demonstrated that symptoms in jarl $(74.7 \%), N a h G(71.7 \%)$, and sid2 $(74.6 \%)$ were more serious than those in wild-type (WT) plants $(67.1 \%)$. The bacterial population, in DC3000-infected leaves, of jarl, NahG, and sid2 was 1.9-, 2.8-, and 3.0-fold higher than WT, respectively, consistent with the observed infection phenotype (Fig. 2C). These results indicate that both SA and JA pathways play essential roles in Arabidopsis-DC3000 interactions. Furthermore, DC3000 accumulations in NahG and sid2 were higher than in jarl, implying that the SA pathway may play a
A

Mock
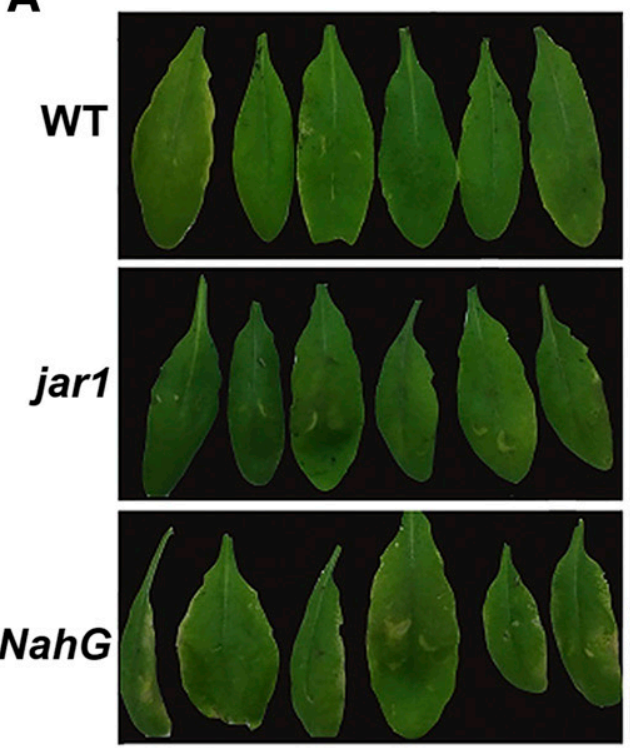

NahG

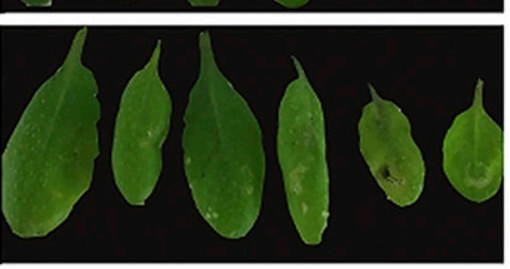

B

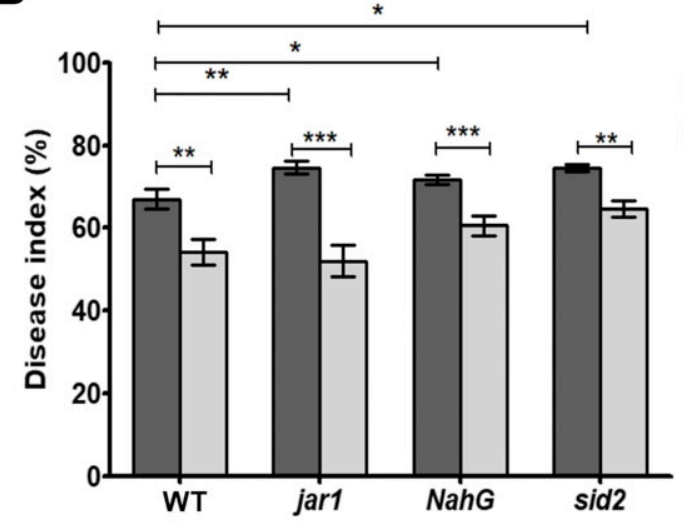

Mock+Pst
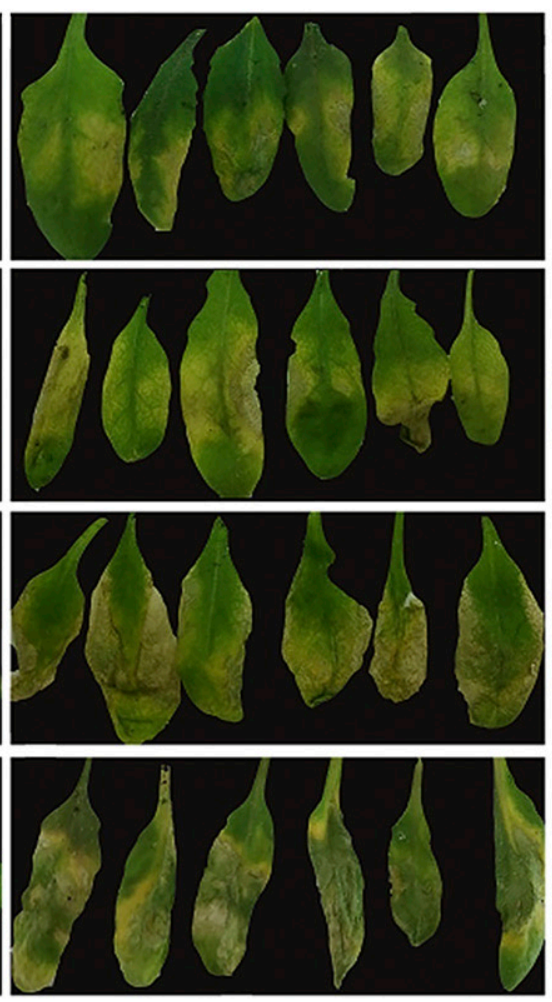
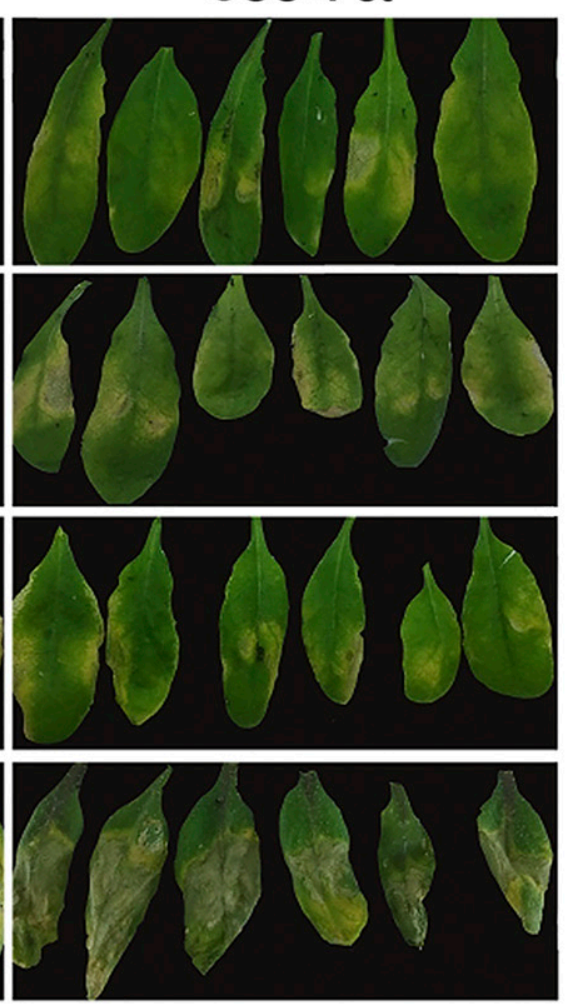

C
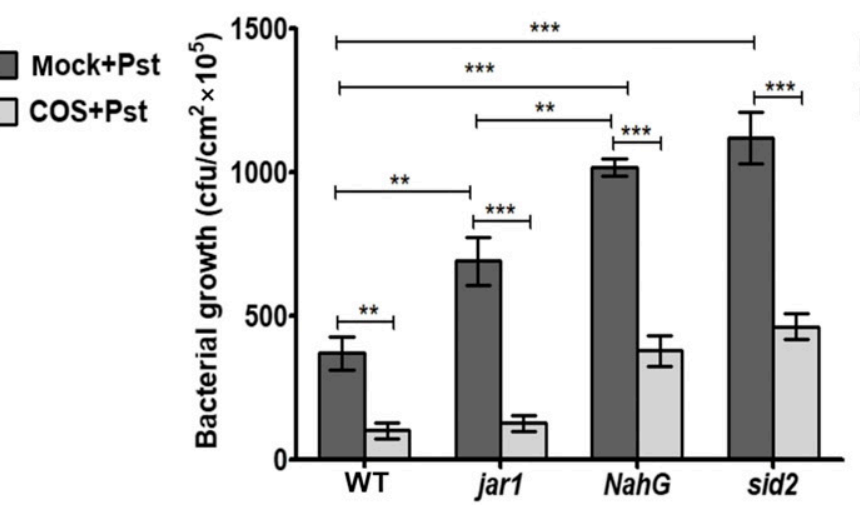

Mock+Pst

cos+Pst

Fig. 2. The function of chitosan oligosaccharide (COS) in the Arabidopsis-Pseudomonas syringae pv. tomato DC3000 interaction and disease control effect on different mutants. Arabidopsis pretreated with water, then inoculated with $10 \mathrm{mM} \mathrm{MgSO}$ is noted as Mock. Arabidopsis pretreated with water or $50 \mathrm{mg}$ of COS per liter, then inoculated with DC3000 are noted as Mock + Pst or COS + Pst, respectively. Each group contained more than 30 Arabidopsis plants. A, Disease symptoms of Arabidopsis leaves caused by DC3000 infection. The representative infected leaves from each treatment were photographed at 4 days after inoculation with DC3000. B, The disease index evaluating the disease symptoms on DC3000-infected leaves. C, Bacterial growth assay of DC3000 in per square centimeter of leaf tissues. Values are the means \pm standard deviation in three independent measurements. One asterisk $(*)$ indicates significant difference at $P<0.05$, two (**) at $P<0.01$, and three (***) at $P<0.001$. 
more important role in Arabidopsis-DC3000 interaction than the JA pathway. Since the two SA-deficient mutants, NahG and sid2, showed a similar trend in Arabidopsis-DC3000 interaction, we choose to focus on $N a h G$ for further investigation.

The expression of $a v r P t o B$ and $r e c A$ in inoculated leaves was highly consistent with the bacterial populations. The expression of avrPtoB in jarl and NahG was 13.1- and 59.5-fold higher than in WT, respectively (Fig. 3A). Additionally, recA expression in jarl and $N a h G$ was 3.8- and 50.3-fold higher than in WT, respectively (Fig. 3B). These quantitative polymerase chain reaction (qPCR) data further confirmed that both SA- and JA-mediated pathways are important for Arabidopsis immune response against DC3000 infection, and, moreover, the SA pathway appears to play a more critical role in the interaction.

\section{COS pretreatment induces resistance to $\mathrm{DC3000}$} in SA- or JA-deficient mutants.

We investigated the involvement of SA and JA pathways in COS-induced resistance to DC3000 in Arabidopsis by using jarl, NahG, and sid2 mutants. WT plants and the different signaling pathway-deficient mutants were pretreated with a 50 -mg/liter concentration of COS 3 days before DC3000 inoculation in the COS $+P s t$ group, and the symptoms of inoculated leaves were significantly reduced compared with the Mock $+P s t$ group (pretreated with water 3 days before DC3000 inoculation) (Fig. 2A). Additionally, the disease index of WT, jarl, NahG, and sid2 plants in the COS + Pst group decreased by $19.2,30.3,15.4$, and $13.1 \%$, respectively, compared with the Mock $+P s t$ group (Fig. 2B). Further, the amount of DC3000
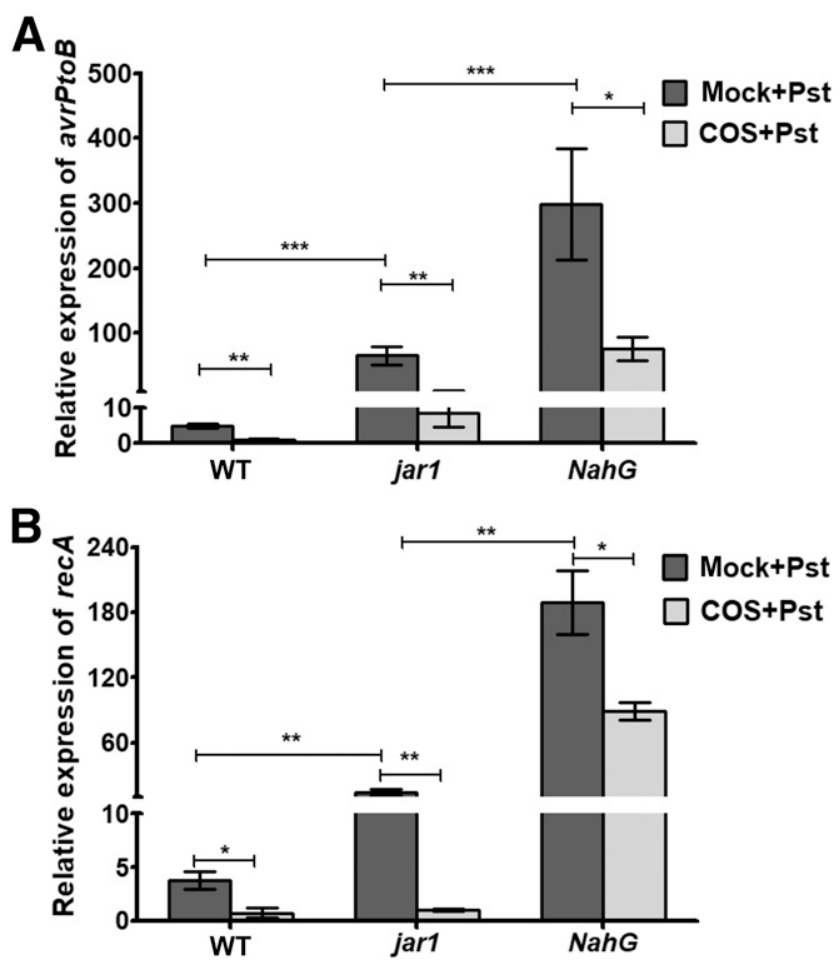

Fig. 3. A, The expression of the marker genes of Pseudomonas syringae pv tomato DC3000 in infected leaves avrPtoB and B, recA. Arabidopsis pretreated with water or $50 \mathrm{mg}$ of chitosan oligosaccharide (COS) per liter, then inoculated with DC3000 are noted as Mock + Pst or COS + Pst, respectively. Each group contained more than 30 Arabidopsis plants. The expression values of the individual genes were normalized using actin 8 as an internal standard. Values are the means \pm standard deviation from three technical replicates. Similar results were obtained in three biological replicates. One asterisk $(*)$ indicates significant difference at $P<0.05$, two (**) at $P<0.01$, and three $(* * *)$ at $P<0.001$. bacteria in WT, jarl, NahG, and sid2 in COS-pretreated inoculated leaves was reduced sharply compared with the Mock+ Pst group, decreasing by $72.7,81.7,62.8$, and $58.7 \%$, respectively (Fig. 2C). These results suggest that COS induces resistance to DC3000 in SA- and JA-deficient mutants.

The expression of $a v r P t o B$ and $r e c A$ demonstrate similar trends. In the COS-pretreated group, the expression of $a v r P t o B$ was reduced by $80.0,87.2$, and $74.4 \%$, and $r e c A$ expression was reduced by $80.3,93.0$, and $52.8 \%$, respectively, in WT, jarl, and $N a h G$ compared with the Mock + Pst group (Fig. 3A and B). COS induced resistance is less effectively in NahG than in either WT or jarl, further confirmed that SA-mediated pathway plays more critical role than JA pathway in DC3000 resistance.

COS pretreatment induced resistance in WT, jarl, and also, although to a lesser degree, in NahG and sid 2 plants, implying that SA- and JA-mediated pathways play redundant roles in COS-induced resistance.

\section{COS pretreatment enhances the expression} of SA- and JA-related genes in Arabidopsis.

To investigate the status of the SA and JA signaling pathways in COS-pretreated Arabidopsis plants, the expression of SA $(P R 1, P R 2$, and PR5) and JA (PDF1.2 and VSP2) pathwayrelated genes were detected.

In WT plants, the expression of $P R 1, P R 2$, and $P R 5$ in COSpretreated inoculated plants were 3.6, 42.4, and 34.7-fold higher than in the Mock + Pst group, respectively, suggesting that the SA signaling pathway is highly activated in COSpretreated plants (Fig. 4 A to C). Moreover, JA signaling pathway-related genes were also induced, the expression of $P D F 1.2$ and $V S P 2$ in the COS+Pst group were enhanced 52.5 and 22.4-fold compared with the Mock $+P$ st group (Fig. 5A and B). Thus, COS induced resistance to DC3000 in WT by activating both SA and JA-mediated pathways.

In jarl, the SA signaling pathway-related genes $(P R 1, P R 2$, and PR5) were upregulated $10.0,16.9$, and 25.3 -fold, respectively, in COS-pretreated plants as compared with the Mock $+P s t$ group (Fig. 4A to C). COS pretreatment did not show similar upregulation in PDF1.2 and VSP2 in the jarl mutant, due to the blocked JA pathway (Fig. 5A and B). Thus, COS induces resistance to DC3000 in jarl by activating the SA-dependent signaling pathway.

Furthermore, in the jarl mock group $($ Mock $+P s t)$, the expression of $P R 1, P R 2$, and $P R 5$ was $3.5,11.2$, and 5.1-fold higher, respectively, than in the WT Mock group. This implies that the SA signaling pathway was activated in jarl due to the blocked JA pathway. Moreover, after COS pretreatment (COS+ $P$ st group), $P R 1, P R 2$, and $P R 5$ expression in jarl was 9.9, 4.5, and 3.7-fold higher than in WT. The highly activated SA signaling pathway might be responsible for the reduced DC3000 infection in COS-pretreated jarl plants compared with WT. A highly activated SA pathway in jarl was enough for a full response of COS-induced resistance to DC3000, thus, the JA pathway plays a redundant role.

COS pretreatment highly enhanced the expression of SA signaling pathway-related genes in WT and jarl but not in $N a h G$ (Fig. 4A to C). NahG plants have an introduced bacterial $n a h G$ gene that encodes salicylate hydroxylase, which degrades SA; thus, NahG plants accumulate very little amount of SA. Therefore, COS pretreatment has no induction effect on $P R 1$, $P R 2$, and $P R 5$ in NahG. However, the expression of PDF1.2 and VSP2 was upregulated by 1130.0- and 12.4-fold, respectively, in the COS pretreatment group $(\mathrm{COS}+P s t)$ compared with the Mock+Pst group (Fig. 5A and B). Thus, COS induces resistance to DC3000 in NahG by activating the JA-dependent signaling pathway, revealing the redundant role of SA pathway in COS-induced resistance to DC3000. 
Moreover, expression of both PDF1.2 and VSP2 was higher (47.0- and 2.8-fold, respectively) in the $N a h G$ mock group compared with WT and, additionally, was upregulated (1,010.4and 1.6-fold, respectively) in the COS-pretreated group compared with the same treatment group in WT. These results suggest that the JA pathway is highly activated in NahG. However, in contrast to jarl, COS pretreatment was less effective at reducing DC3000 infection in $N a h G$, further confirming that the SA pathway plays a more critical role than the JA pathway in DC3000 resistance.

\section{COS pretreatment increases SA- and JA-related protein content in Arabidopsis.}

To verify the qPCR results, the SA- and JA-related proteins NPR 1 and COl1 were detected by Western blot (WB). NPR1 is a key positive regulator of the SA signaling pathway, and the activation by SA usually induces the expression of pathogenesis related genes (Mou et al. 2003; Niu et al. 2016). The WB results demonstrate that following DC3000 inoculation, COS pretreatment significantly enhanced NPR1 content in leaves of WT and jarl, as compared with the mock group, implying an activated SA-dependent signaling pathway (Fig. 6A).

As expected, this protein content increase after COS pretreatment did not occur in $N a h G$, although the basal protein content of NPR1 is much higher than in WT and jarl, possibly due to a highly activated JA signaling pathway in the $N a h G$ mutant. It is generally accepted that NPR1 plays a complex role in SA and JA pathway crosstalk, and evidence is accumulating that nuclear-localized NPR1 regulates the SA-mediated defense response, whereas cytosolic NPR1 is required for JA-dependent pathway activation (Nie et al. 2017; Pieterse et al. 2014; Ramírez et al. 2010). The higher basal level of NPR1 in NahG may be due to an overactivated JA signaling pathway accumulating cytosolic NPR1.

COl1 is an essential component of the JA receptor, and its content varies depending on JA levels (Wasternack and Hause 2013; Xie et al. 1998). As expected, COl1 content in the COS+ Pst group is enhanced in WT and NahG compared with the Mock + Pst group; however, there was no significant increase in jarl, suggesting that the JA signaling pathway is activated after COS pretreatment in WT and NahG (Fig. 6B). Basal amounts of CO11 in jarl are lower than that in WT and NahG, due to an impaired JA biosynthesis pathway in jarl.

\section{COS pretreatment upregulates SA and JA levels in Arabidopsis.}

To further confirm the qPCR data and WB results, we measured the SA and JA content by liquid chromatography-mass spectrometry (LC-MS) in the different Arabidopsis genotypes under different treatments.

In the mock group without DC3000 infection, the basal SA content in jarl was 1.5-fold higher than WT, implying the highly activated SA pathway in jarl. The basal SA was 2.2-fold lower, and JA content was 4.2-fold higher in NahG, respectively, compared with the WT mock groups, suggesting the suppressed SA pathway and enhanced JA pathway in $N a h G$.

And as expected, the COS pretreatment significantly enhanced the SA and JA content in WT by 1.8- and 1.4-fold, respectively, compared with the mock treatment group (Mock+ Pst). Furthermore, COS pretreatment also increased SA content in jarl (by 1.9-fold) and JA content in NahG (by 1.3-fold) (Fig. $7 \mathrm{~A}$ and $\mathrm{B}$ ). These results further revealing that both SA- and JA-mediated signaling pathways participate in COS-induced resistance to DC3000 in Arabidopsis.

\section{DISCUSSION}

COS is an effective plant immunity elicitor that is able to induce resistance to pathogens during infection in various plants, according to previous studies. This includes tobaccoTMV, Arabidopsis-TMV, tomato-Botrytis cinerea, grapevinePlasmopara viticola, and rice-SRBSDV (Jia et al. 2016; Yang et al. 2017; Yin et al. 2010; Zhang and Chen 2009). As MAMPs, the perception of COS by the host cell leads to the activation of a complicated signaling network, including the transient accumulation of signal molecules such as $\mathrm{Ca}^{2+}, \mathrm{NO}$, and $\mathrm{H}_{2} \mathrm{O}_{2}$, the upregulation of the mitogen-activated protein kinase signaling cascade pathway, and activation of the SA and JA-mediated signaling pathways (Jia et al. 2016; Poncini et al. 2017; Yang et al. 2017; Yin et al. 2013). The COS induction mechanism is very complex and requires further investigation.

In this study, the potential defense signaling pathways in COS-induced resistance to DC3000 were investigated by using WT, jarl, NahG, and sid2. We found that both SA- and
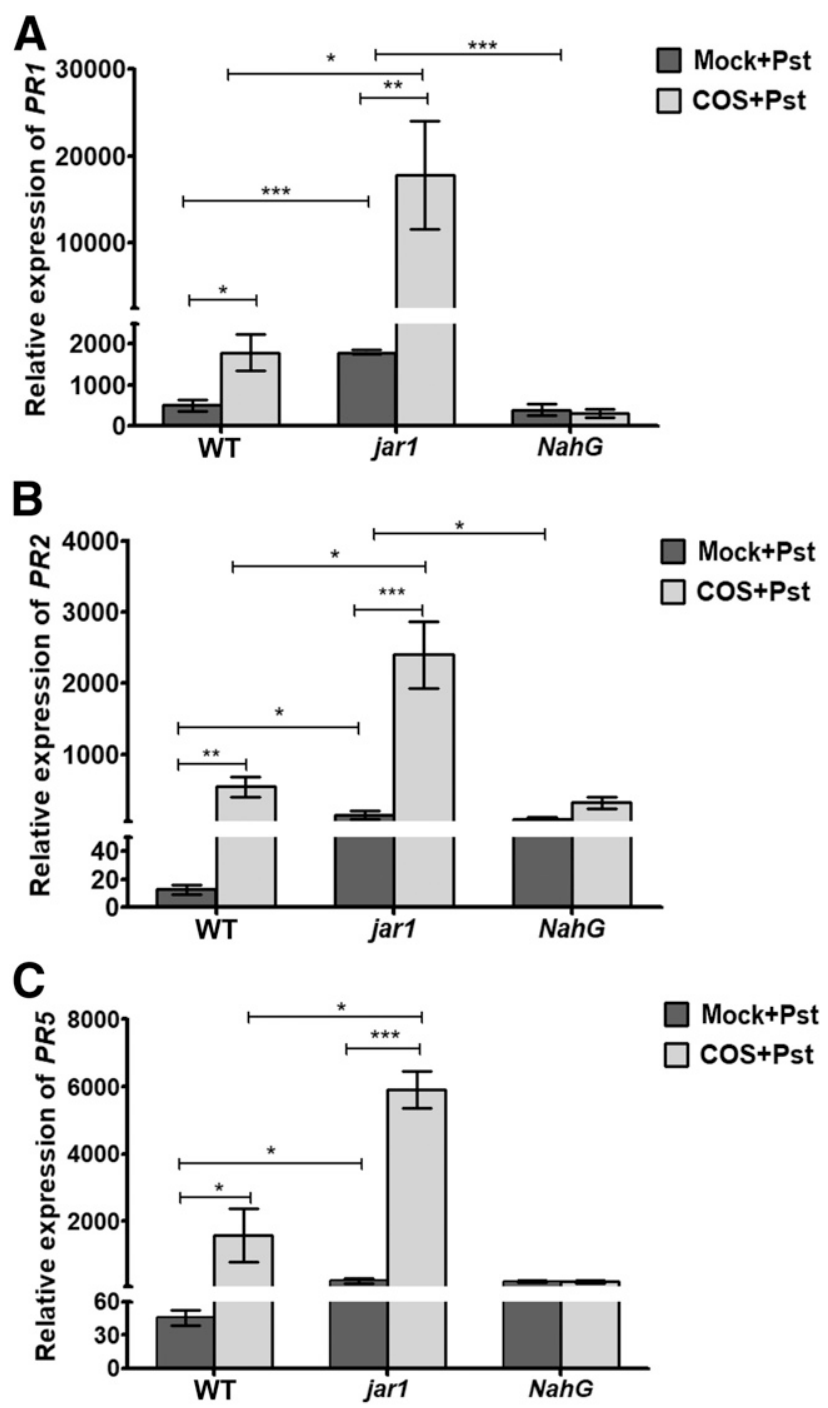

Fig. 4. A, The expression of salicylic acid-mediated pathway related genes PR1, B, PR2, and C, PR5. Arabidopsis pretreated with water or $50 \mathrm{mg}$ of chitosan oligosaccharide (COS) per liter, then inoculated with Pseudomonas syringae pv. tomato DC3000 are noted as Mock + Pst or COS + Pst, respectively. Each group contained more than thirty Arabidopsis plants. The expression values of the individual genes were normalized using actin 8 as an internal standard, then compared with mock-treated wild type (WT). Values are the means \pm standard deviation from three technical replicates. Similar results were obtained in three biological replicates. One asterisk $(*)$ indicates significant difference at $P<0.05$, two $(* *)$ at $P<0.01$, and three (***) at $P<0.001$. 
JA-mediated pathways play positive roles in reducing infection in Arabidopsis after DC3000 inoculation. The crosstalk between SA and JA signaling pathways is complex, and they influence each other through synergistic and antagonistic interactions. In many cases, SA and JA signaling pathways act antagonistically. For example, activation of SA biosynthesis will inhibit JA biosynthesis or signaling in many plant-pathogen interactions (Thaler et al. 2012; Zhang et al. 2015). In Arabidopsis-TMV or tobacco-TMV interactions, SA functions as a positive regulator of TMV resistance. According to previous studies, TMV infection or COS pretreatment enhanced SA content while decreasing JA accumulation (Jia et al. 2016).

However, in Arabidopsis-DC3000 interaction, both SA and JA signaling pathways were activated; thus, they may work in independent or cooperative manners without obvious antagonism. Betsuyaku et al. (2018) found that, although the SA and JA pathways are both activated in the ArabidopsisDC3000 interaction, they are active in different domains around the infection site. While SA accumulates in the cells directly around DC3000 infection sites, the JA pathway is activated in cells surrounding SA-active cells (Betsuyaku et al. 2018).

DC3000 resistance was more obviously impaired in NahG and sid2 than jarl, revealing the more critical role of the SAmediated pathway than JA pathway in Arabidopsis defense to DC3000. According to previous studies, virulence systems of DC3000 usually promote infection by targeting the JA signaling pathway. For example, DC3000 is able to synthesize the polyketide toxin coronatine, which structurally mimics JA,

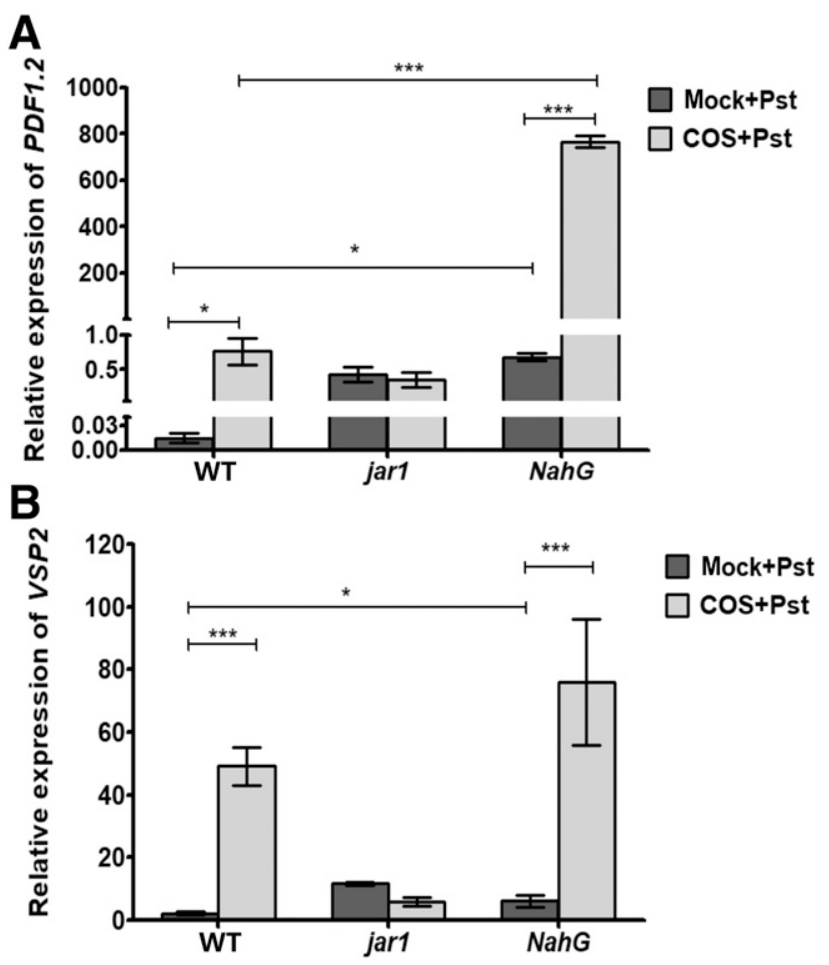

Fig. 5. A, The expression of jasmonic acid-mediated signaling pathwayrelated genes PDF1.2 and B, VSP2. Arabidopsis pretreated with water or 50 $\mathrm{mg}$ of chitosan oligosaccharide (COS) per liter, then inoculated with Pseudomonas syringae pv. tomato DC3000 are noted as Mock + Pst or COS + Pst, respectively. Each group contained more than thirty Arabidopsis plants. The expression values of the individual genes were normalized using actin 8 as an internal standard, then compared with mock-treated wild type (WT). Values are the means \pm standard deviation from three technical replicates. One asterisk $(*)$ indicates significant difference at $P<0.05$, two $(* *)$ at $P<0.01$, and three $(* * *)$ at $P<0.001$. interfering with the JA-mediated signaling pathway (Staswick and Tiryaki 2004; Xin and He 2013; Zhao et al. 2003). This may be responsible for the major role of the SA signaling pathway in limiting DC3000 infection in Arabidopsis.

COS pretreatment activated both SA- and JA-mediated pathways in WT Arabidoposis, which all worked positively in Arabidopsis defense to DC3000. However, SA and JA pathways play redundant roles in COS-induced resistance to DC3000 in SA or JA pathway-deficient mutants due to the highly activated JA or SA pathway after COS pretreatment. This induction effect of COS that activates different signaling pathways under different situations makes COS work effectively in multiple plant-pathogen interactions. This phenomenon also appeared in other elicitor-treated plants, according to previous studies, such as plant growth-promoting fungus Penicillium simplicissimum GP17-2 (Hossain et al. 2007) and Phoma sp. strain GS8-1(Hossain et al. 2008). GP17-2 or GS8-1 pretreatment were fully protective against DC3000 in jarl, similar to WT, and also worked effectively in NahG and $n p r l$, although to a lesser degree. Furthermore, treatment with GP172 or GS8-1 resulted in enhanced expression of SA- and JArelated genes and activated both SA and JA pathways in WT, just like COS-induced resistance.

Generally, the SA signaling pathway is associated with biotroph resistance, whereas the JA/ET signaling pathway is involved in defending against necrotrophs (Robert-Seilaniantz et al. 2007). COS-induced resistance in the ArabidopsisDC3000 (hemibiotrophic pathogen) interaction is mainly achieved by activating both SA- and JA-mediated pathways. This is quite different from Arabidopsis-TMV (biotrophic pathogen) interactions, since COS only activates the SA signaling pathway (Jia et al. 2016). Further, in tomato, rice, and rapeseed, COS pretreatment activates the JA-dependent signaling pathway, which results in resistance to necrotrophic pathogens (Doares et al. 1995; Rakwal et al. 2002; Yin et al. 2013). Based on these results, COS pretreatment activates different defense pathways in different plants under infection by different pathogens, activating one or both the SA-dependent and JA-dependent, signaling pathways. Thus, COS functions as an effective and broad-spectrum plant immunity elicitor in multiple plant-pathogen interactions.

The broad-spectrum disease control effects demonstrated by COS pretreatment in different plant-pathogen interactions are ubiquitous among plant immunity elicitors. For example, the well-studied pathogen-associated molecular pattern flg22 was found to induce resistance in Arabidopsis against DC3000 by

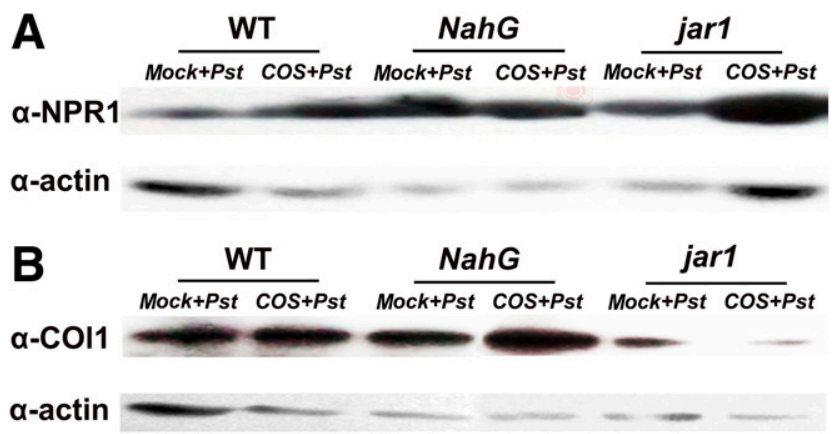

Fig. 6. A, The amount of salicylic acid- and jasmonic acid-mediated pathway related proteins, NPR1and B, COl1. Arabidopsis pretreated with water or $50 \mathrm{mg}$ of chitosan oligosaccharide (COS) per liter, then inoculated with Pseudomonas syringae pv. tomato DC3000 are noted as Mock + Pst or COS + Pst, respectively. This was a representative experiment and was independently repeated three times using different samples from independent treatment. 
activating both SA- and JA-dependent signaling pathways (Navarro et al. 2004; Wang et al. 2018). Additionally, flg22 induced resistance to Golovinomyces cichoracearum in Arabidopsis by activating the SA signaling pathway (Lu et al. 2015). In addition to flg22, other plant immunity regulators, such as harpin, Bacillus cereus AR156, and plant growth-promoting fungi also showed similar induction responses (Chuang et al. 2014; Hossain et al. 2008; Jiang et al. 2016; Nie et al. 2017; Wang et al. 2018).

Certainly, signaling pathways in addition to SA and JA pathways may also be involved in COS-induced resistance. COS induction mechanism is complex needs further investigation.

\section{MATERIALS AND METHODS}

Plant materials and treatment.

COS was obtained from Dalian GlycoBio Co. Ltd. (Dalian, China), with a degree of polymerization from 2 to 10 and a degree of deacetylation of $95 \%$. Arabidopsis ecotype Columbia (Col-0, WT) and mutant lines, NahG, sid2 (SA-

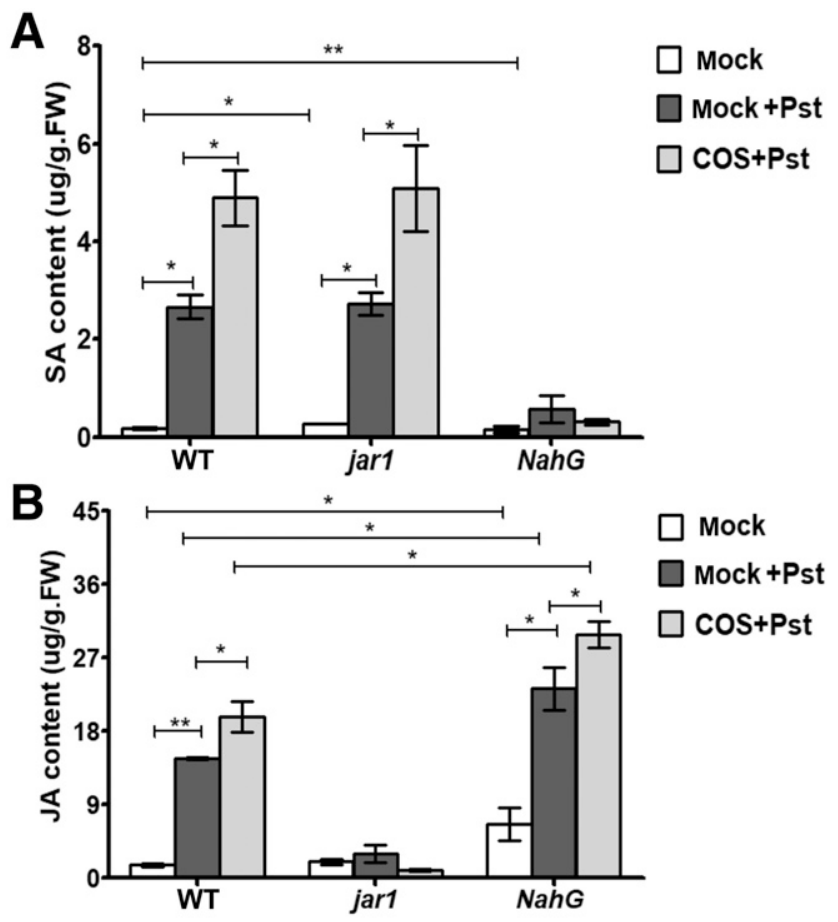

Fig. 7. A, The relative level of salicylic acid (SA) and B, jasmonic acid (JA) in different mutants. Arabidopsis pretreated with water, then inoculated with $10 \mathrm{mM} \mathrm{MgSO}_{4}$ is noted as Mock. Arabidopsis pretreated with water or $50 \mathrm{mg}$ of COS per liter, then inoculated with DC3000 are noted as Mock + Pst or COS + Pst, respectively. Values were presented as means \pm standard deviation from three independent measurements. One asterisk $(*)$ indicates significant difference at $P<0.05$, two (**) at $P<0.01$, and three $(* * *)$ at $P<0.001)$. deficient mutants), and jarl (JA-deficient mutant) were used for experiments.

Arabidopsis plants were cultivated as described previously (Jia et al. 2016). Thirty-day-old plants, grown in soil, were used for experiments. To investigate the optimal COS pretreatment time, WT plants were sprayed with a 50-mg/liter COS solution at 3,2, and 1 days before and $0,1,2$, and 3 days after DC3000 inoculation. Plants inoculated with DC3000 without any COS treatment served as control.

WT, jarl, NahG, and sid2 plants were evenly assigned into either the Mock group (spraying water 3 days before inoculating with $10 \mathrm{mM} \mathrm{MgSO}_{4}$ ), the Mock + Pst group (spraying water 3 days before inoculating with DC3000), or the COS $+P s t$ group (spraying a $50 \mathrm{mg} /$ liter COS solution 3 days before inoculating with DC3000). Each treatment group contained at least 30 plants, and each experiment was repeated at least three times. The plants were inoculated with DC3000 according to the method described as below.

\section{DC3000 inoculation.}

DC3000 was grown in King's B medium (per milliliter, $10 \mathrm{mg}$ of protease peptone, $1.5 \mathrm{mg}$ of $\mathrm{K}_{2} \mathrm{HPO}_{4}, 15 \mathrm{mg}$ of glycerol) supplemented with $25 \mathrm{mg}$ of rifampicin per milliliter, at $28^{\circ} \mathrm{C}$. Rosette leaves of Arabidopsis plants were inoculated with $10 \mathrm{mM} \mathrm{MgSO}_{4}$ or DC3000 suspensions in $10 \mathrm{mM} \mathrm{MgSO}_{4}$, using a 1-ml syringe (without a needle). The bacterial dose infiltrated into the leaves was $1 \times 10^{5} \mathrm{CFU}$ per square centimeter of leaf area (equivalent to an optical density at $600 \mathrm{~nm}=0.002$ ).

According to the proportion (S) of necrotic lesion area on leaves, the symptoms of infected leaves were divided into four levels: $1(0<\mathrm{S}<0.25), 2(0.25<\mathrm{S}<0.5), 3(0.5<\mathrm{S}<0.75)$, and $4(0.75<\mathrm{S}<1)$. The symptom data were obtained 4 days after DC3000 infection, using the following formula: Disease index $(\%)=\left(\sum(\right.$ level $\times$ leaves in the level $) \div$ total leaves $\div$ the highest level $) \times 100$. Inoculated leaves were collected at 4 days after inoculation, were weighed, were quickly frozen in liquid nitrogen, and were kept in $-80^{\circ} \mathrm{C}$ for further experiments.

\section{Gene expression analysis by qPCR.}

Total RNA was extracted using a TRIzol reagent kit (Lablead) and was quantified by the Scan Drop100 (Analytik Jena AG). Equal amounts of RNA were reverse transcribed into cDNA with a RevertAid first-strand cDNA synthesis kit (Thermo Fisher Scientific). The diluted newly transcribed cDNA was used for gene expression detection by qPCR (qTOWER 2.2, Analytik Jena AG). The reaction volumes were $20 \mu \mathrm{l}$, consisting of cDNA dilutions, qPCR MasterMix, and genespecific primers (Table 1). The gene expression data were calculated by using the $2^{-\Delta \Delta C t}$ method (Livak and Schmittgen 2001).

\section{WB detection.}

Plant protein extraction and sodium dodecyl sulfatepolyacrylamide gel electrophoresis (SDS-PAGE) were carried out as described previously (Jia et al. 2016). Total protein $(50 \mu \mathrm{g})$ was loaded into the SDS-PAGE (10\%) gels. Separated

Table 1. Primers used for quantitative polymerase chain reaction

\begin{tabular}{|c|c|c|c|}
\hline Gene & Locus ID & Forward & Reverse \\
\hline actin8 & AT1G49240 & CACATGCTATCCTCCGTCTC & CACTTGTCCGTCGGGTAAT \\
\hline recA & - & CGGCAAGGGTATCTACCTCA & CTTTGCAGATTTCCGGGTTA \\
\hline avrPtoB & - & CACTTGTCCGTCGGGTAAT & CATGAACGCCAGGTCCTTAT \\
\hline PRI & AT2G14610 & AATGCTCAAGATAGCCCACAAG & AATAAGTCACCGCTACCCCAG \\
\hline$P R 2$ & AT3G57260 & AAAACGGAGGCCGACAAG & CGGAGGAGACGTATCAGTGGT \\
\hline PR5 & AT1G75040 & ATTGACTCCAGGTGCTTCCC & GCCGTAGAAATCTTTGCCG \\
\hline PDF1.2 & AT5G44420 & TCACCCTTATCTTCGCTGCTC & ATGTCCCACTTGGCTTCTCG \\
\hline VSP2 & AT2G06530 & TGTGAACAGGCAGATGAACC & GCGATACCGATCTCGTCAA \\
\hline
\end{tabular}


proteins were transferred to polyvinylidene difluoride membranes, blocked with $5 \%$ (wt/vol) nonfat dry milk in TBST buffer $(20 \mathrm{mM}$ Tris- $\mathrm{HCl}, \mathrm{pH} 8.0$, and $150 \mathrm{mM} \mathrm{NaCl}, 0.05 \%$ Tween 20) for 3 to $4 \mathrm{~h}$ at room temperature.

Membranes were cut into two parts at $55 \mathrm{kDa}$, were probed with different antibodies, including NPR1 (regulatory protein, $66 \mathrm{kDa}$ ), COl1 (coronate insensitive 1, $67 \mathrm{kDa}$ ), and plant actin $(40 \mathrm{kDa})$. The blot was incubated in the primary antibody of NPR1 or COl1 (Agrisera), at a dilution of 1:500, overnight at $4^{\circ} \mathrm{C}$, followed by washing with TBST buffer three times. The blot was then incubated with the secondary antibody, HRP AffiniPure goat antirabbit immunoglobulin $\mathrm{G}$ (IgG) (Earthox), at a dilution of 1:1,000, for $2 \mathrm{~h}$ at room temperature. For plant actin detection, the membrane was probed with antiplant actin mouse monoclonal antibody $(1: 2,000)$ (Earthox) and HRP AffiniPure goat antirabbit IgG $(1: 2,000)$ (Earthox) as a secondary antibody.

\section{SA and JA quantification by LC-MS.}

The extraction and quantification of SA and JA in infected leaf samples were carried out by using the LC-MS method described previously with some modification (de Sá et al. 2014). SA and JA standards were obtained from Sigma. Chromatography conditions were modified using a hypercarb column (Thermo Fisher) $(150 \times 2.1 \mathrm{~mm}, 5 \mu \mathrm{m})$ at $35^{\circ} \mathrm{C}$, and an elution gradient of $3 \mathrm{ml}$ of formic acid per liter, adjusted to $\mathrm{pH} 9.0$ with ammonium hydroxide (A) and acetonitrile (B) for $20 \mathrm{~min}$ at a flow rate of $0.2 \mathrm{ml} / \mathrm{min}$. The details of gradient conditions were as follows: $0 \mathrm{~min}, 90 \% \mathrm{~A}+10 \% \mathrm{~B} ; 10 \mathrm{~min}, 40 \% \mathrm{~A}+60 \%$ B; $14 \min , 40 \% \mathrm{~A}+60 \% \mathrm{~B} ; 14.1 \mathrm{~min}, 90 \% \mathrm{~A}+10 \% \mathrm{~B}$; and 20 min, 90\% A + 10\% B. Q-trap 5500 (Ab Sciex) with optimized collision energy was used for SA and JA detection.

\section{ACKNOWLEDGMENTS}

We are grateful to J. Zhou (Institute of Genetics and Developmental Biology, Chinese Academy of Sciences) and L. Hao (Shenyang Normal University, China) for kindly providing the Pseudomonas syringae pv. tomato DC3000 and SA-deficient mutant sid2, respectively. Many thanks for the help of P. Howlader in grammar modification.

\section{LITERATURE CITED}

Abramovitch, R. B., and Martin, G. B. 2005. AvrPtoB: A bacterial type III effector that both elicits and suppresses programmed cell death associated with plant immunity. FEMS Microbiol. Lett. 245:1-8.

Betsuyaku, S., Katou, S., Takebayashi, Y., Sakakibara, H., Nomura, N., and Fukuda, H. 2018. Salicylic acid and jasmonic acid pathways are activated in spatially different domains around the infection site during effectortriggered immunity in Arabidopsis thaliana. Plant Cell Physiol. 59:8-16.

Chien, C. F., Mathieu, J., Hsu, C. H., Boyle, P., Martin, G. B., and Lin, N. C. 2013. Nonhost resistance of tomato to the bean pathogen Pseudomonas syringae pv. syringae $\mathrm{B} 728 \mathrm{a}$ is due to a defective E3 ubiquitin ligase domain in avrptobb $728 \mathrm{a}$. Mol. Plant-Microbe Interact. 26:387-397.

Chuang, H. W., Chang, P. Y., and Syu, Y. Y. 2014. Harpin protein, an elicitor of disease resistance, acts as a growth promoter in Phalaenopsis orchids. J. Plant Growth Regul. 33:788-797.

Cuppels, D. A. 1986. Generation and characterization of Tn5 insertion mutations in Pseudomonas syringae pv. tomato. Appl. Environ. Microbiol. 51:323-327.

de Sá, M., Ferreira, J. P., Queiroz, V. T., Vilas-Boas, L., Silva, M. C., Almeida, M. H., Guerra-Guimarães, L., and Bronze, M. R. 2014. A liquid chromatography/electrospray ionisation tandem mass spectrometry method for the simultaneous quantification of salicylic, jasmonic and abscisic acids in Coffea arabica leaves. J. Sci. Food Agric. 94: 529-536.

Desaki, Y., Miyata, K., Suzuki, M., Shibuya, N., and Kaku, H. 2018. Plant immunity and symbiosis signaling mediated by LysM receptors. Innate Immun. 24:92-100

Doares, S. H., Syrovets, T., Weiler, E. W., and Ryan, C. A. 1995. Oligogalacturonides and chitosan activate plant defensive genes through the octadecanoid pathway. Proc. Natl. Acad. Sci. U.S.A. 92:4095-4098.
Hadwiger, L. A. 2015. Anatomy of a nonhost disease resistance response of pea to Fusarium solani: PR gene elicitation via DNase, chitosan and chromatin alterations. Front. Plant Sci. 6:373.

Hadwiger, L. A., and Tanaka, K. 2017. Non-host resistance: DNA damage is associated with SA signaling for induction of PR genes and contributes to the growth suppression of a pea pathogen on pea endocarp tissue. Front. Plant Sci. 8:446.

Hossain, M. M., Sultana, F., Kubota, M., Koyama, H., and Hyakumachi, M. 2007. The plant growth-promoting fungus Penicillium simplicissimum GP17-2 induces resistance in Arabidopsis thaliana by activation of multiple defense signals. Plant Cell Physiol. 48:1724-1736.

Hossain, M. M., Sultana, F., Kubota, M., Koyama, H., and Hyakumachi, M. 2008. Systemic resistance to bacterial leaf speck pathogen in Arabidopsis thaliana induced by the culture filtrate of a plant growth-promoting fungus (PGPF) Phoma sp GS8-1. J. Gen. Plant Pathol. 74:213-221.

Jia, X., Meng, Q., Zeng, H., Wang, W., and Yin, H. 2016. Chitosan oligosaccharide induces resistance to Tobacco mosaic virus in Arabidopsis via the salicylic acid-mediated signalling pathway. Sci. Rep. 6:26144.

Jiang, C. H., Fan, Z. H., Xie, P., and Guo, J. H. 2016. Bacillus cereus AR156 extracellular polysaccharides served as a novel micro-associated molecular pattern to induced systemic immunity to Pst DC3000 in Arabidopsis. Front. Microbiol. 7:664.

Jovanovic, M., Waite, C., James, E., Synn, N., Simpson, T., Kotta-Loizou, I., and Buck, M. 2017. Functional characterization of key residues in regulatory proteins $\mathrm{HrpG}$ and $\mathrm{HrpV}$ of Pseudomonas syringae pv. tomato DC3000. Mol. Plant-Microbe Interact. 30:656-665.

Katagiri, F., Thilmony, R., and He, S. Y. 2002. The Arabidopsis thaliana-Pseudomonas syringae interaction. Arabidopsis Book 1:e0039.

Khare, E., Kim, K., and Lee, K. J. 2016. Rice OsPBL1 (ORYZA SATIVA ARABIDOPSIS PBS1-LIKE 1) enhanced defense of Arabidopsis against Pseudomonas syringae DC3000. Eur. J. Plant Pathol. 146: 901-910.

Kloek, A. P., Verbsky, M. L., Sharma, S. B., Schoelz, J. E., Vogel, J., Klessig, D. F., and Kunkel, B. N. 2001. Resistance to Pseudomonas syringae conferred by an Arabidopsis thaliana coronatine-insensitive (coil) mutation occurs through two distinct mechanisms. Plant J. 26 509-522.

Koornneef, A., and Pieterse, C. M. J. 2008. Cross talk in defense signaling. Plant Physiol. 146:839-844.

Kouzai, Y., Kimura, M., Watanabe, M., Kusunoki, K., Osaka, D., Suzuki, T., Matsui, H., Yamamoto, M., Ichinose, Y., Toyoda, K., Matsuura, T., Mori, I. C., Hirayama, T., Minami, E., Nishizawa, Y., Inoue, K., Onda, Y., Mochida, K., and Noutoshi, Y. 2018. Salicylic acid-dependent immunity contributes to resistance against Rhizoctonia solani, a necrotrophic fungal agent of sheath blight, in rice and Brachypodium distachyon. New Phytol. 217:771-783.

Kraus, C. M., Munkvold, K. R., and Martin, G. B. 2016. Natural variation in tomato reveals differences in the recognition of AvrPto and AvrPtoB effectors from Pseudomonas syringae. Mol. Plant 9:639-649.

Li, S. J., and Zhu, T. H. 2013. Biochemical response and induced resistance against anthracnose (Colletotrichum camelliae) of camellia (Camellia pitardii) by chitosan oligosaccharide application. For. Pathol. 43:67-76

Liu, D., Jiao, S., Cheng, G., Li, X., Pei, Z., Pei, Y., Yin, H., and Du, Y. 2018. Identification of chitosan oligosaccharides binding proteins from the plasma membrane of wheat leaf cell. Int. J. Biol. Macromol. 111: 1083-1090.

Liu, X., Sun, Y., Korner, C. J., Du, X., Vollmer, M. E., and PajerowskaMukhtar, K. M. 2015. Bacterial leaf infiltration assay for fine characterization of plant defense responses using the Arabidopsis thaliana-Pseudomonas syringae pathosystem. J. Vis Exp. 104:e53364.

Livak, K. J., and Schmittgen, T. D. 2001. Analysis of relative gene expression data using real-time quantitative PCR and the $2^{-\Delta \Delta C(T)}$ method. Methods 25:402-408.

Lu, X., Dittgen, J., Piślewska-Bednarek, M., Molina, A., Schneider, B., Svatoš, A., Doubský, J., Schneeberger, K., Weigel, D., Bednarek, P., and Schulze-Lefert, P. 2015. Mutant allele-specific uncoupling of PENETRATION3 functions reveals engagement of the ATP-binding cassette transporter in distinct tryptophan metabolic pathways. Plant Physiol. 168:814-827.

Mou, Z., Fan, W., and Dong, X. 2003. Inducers of plant systemic acquired resistance regulate NPR1 function through redox changes. Cell 113:935-944.

Naseem, M., Kaltdorf, M., Hussain, A., and Dandekar, T. 2014. The impact of cytokinin on jasmonate-salicylate antagonism in Arabidopsis immunity against infection with Pst DC3000. Plant Signal. Behav. 8: e26791.

Navarro, L., Zipfel, C., Rowland, O., Keller, I., Robatzek, S., Boller, T., and Jones, J. D. 2004. The transcriptional innate immune response to flg22. 
Interplay and overlap with Avr gene-dependent defense responses and bacterial pathogenesis. Plant Physiol. 135:1113-1128.

Nie, P., Li, X., Wang, S., Guo, J., Zhao, H., and Niu, D. 2017. Induced systemic resistance against Botrytis cinerea by Bacillus cereus AR156 through a JA/ET- and NPR1-dependent signaling pathway and activates PAMP-triggered immunity in Arabidopsis. Front. Plant Sci. 8:238.

Niu, D., Wang, X., Wang, Y., Song, X., Wang, J., Guo, J., and Zhao, H. 2016. Bacillus cereus AR156 activates PAMP-triggered immunity and induces a systemic acquired resistance through a NPR1-and SA-dependent signaling pathway. Biochem. Biophys. Res. Commun. 469:120-125.

Pieterse, C. M., Zamioudis, C., Berendsen, R. L., Weller, D. M., Van Wees, S. C., and Bakker, P. A. 2014. Induced systemic resistance by beneficial microbes. Annu. Rev. Phytopathol. 52:347-375.

Poncini, L., Wyrsch, I., Dénervaud Tendon, V., Vorley, T., Boller, T., Geldner, N., Métraux, J. P., and Lehmann, S. 2017. In roots of Arabidopsis thaliana, the damage-associated molecular pattern AtPep1 is a stronger elicitor of immune signalling than flg 22 or the chitin heptamer. PLoS One 12:e0185808.

Rakwal, R., Tamogami, S., Agrawal, G. K., and Iwahashi, H. 2002. Octadecanoid signaling component "burst" in rice (Oryza sativa L.) seedling leaves upon wounding by cut and treatment with fungal elicitor chitosan. Biochem. Biophys. Res. Commun. 295:1041-1045.

Ramírez, V., Van der Ent, S., García-Andrade, J., Coego, A., Pieterse, C. M. J., and Vera, P. 2010. OCP3 is an important modulator of NPR1mediated jasmonic acid-dependent induced defenses in Arabidopsis. BMC Plant Biol. 10:199.

Robert-Seilaniantz, A., Navarro, L., Bari, R., and Jones, J. D. 2007. Pathological hormone imbalances. Curr. Opin. Plant Biol. 10:372-379.

Scalschi, L., Camañes, G., Llorens, E., Fernández-Crespo, E., López, M. M., García-Agustín, P., and Vicedo, B. 2014. Resistance inducers modulate Pseudomonas syringae pv. tomato strain DC3000 response in tomato plants. PLoS One 9:e106429.

Squeglia, F., Berisio, R., Shibuya, N., and Kaku, H. 2017. Defense against pathogens: Structural insights into the mechanism of chitin induced activation of innate immunity. Curr. Med. Chem. 24:3980-3986.

Staswick, P. E., and Tiryaki, I. 2004. The oxylipin signal jasmonic acid is activated by an enzyme that conjugates it to isoleucine in Arabidopsis. Plant Cell 16:2117-2127.

Thaler, J. S., Humphrey, P. T., and Whiteman, N. K. 2012. Evolution of jasmonate and salicylate signal crosstalk. Trends Plant Sci. 17: 260-270.

Todesco, M., Balasubramanian, S., Hu, T. T., Traw, M. B., Horton, M., Epple, P., Kuhns, C., Sureshkumar, S., Schwartz, C., Lanz, C., Laitinen, R. A., Huang, Y., Chory, J., Lipka, V., Borevitz, J. O., Dangl, J. L., Bergelson, J., Nordborg, M., and Weigel, D. 2010. Natural allelic variation underlying a major fitness trade-off in Arabidopsis thaliana. Nature 465:632-636.

Valdés-López, O., Khan, S. M., Schmitz, R. J., Cui, S. Q., Qiu, J., Joshi, T., $\mathrm{Xu}$, D., Diers, B., Ecker, J. R., and Stacey, G. 2014. Genotypic variation of gene expression during the soybean innate immunity response. Plant Genet. Resour-C 12 (S1):S27-S30.

Velásquez, A. C., Oney, M., Huot, B., Xu, S., and He, S. Y. 2017. Diverse mechanisms of resistance to Pseudomonas syringae in a thousand natural accessions of Arabidopsis thaliana. New Phytol. 214:1673-1687.

Wang, M. Y., Chen, Y. C., Zhang, R., Wang, W. X., Zhao, X. M., Du, Y. G., and Yin, H. 2015. Effects of chitosan oligosaccharides on the yield components and production quality of different wheat cultivars (Triticum aestivum L.) in Northwest China. Field Crops Res. 172:11-20.

Wang, S., Zheng, Y., Gu, C., He, C., Yang, M., Zhang, X., Guo, J., Zhao, H., and Niu, D. 2018. Bacillus cereus AR156 activates defense responses to Pseudomonas syringae pv. tomato in Arabidopsis thaliana similarly to flg22. Mol. Plant-Microbe Interact. 31:311-322.
Wasternack, C., and Hause, B. 2013. Jasmonates: Biosynthesis, perception, signal transduction and action in plant stress response, growth and development. An update to the 2007 review in Annals of Botany. Ann. Bot. 111:1021-1058.

Wilson, D. C., Kempthorne, C. J., Carella, P., Liscombe, D. K., and Cameron, R. K. 2017. Age-related resistance in Arabidopsis thaliana involves the MADS-domain transcription factor SHORT VEGETATIVE PHASE and direct action of salicylic acid on Pseudomonas syringae. Mol. Plant-Microbe Interact. 30:919-929.

Xie, D. X., Feys, B. F., James, S., Nieto-Rostro, M., and Turner, J. G. 1998 COI1: An Arabidopsis gene required for jasmonate-regulated defense and fertility. Science 280:1091-1094.

Xin, X. F., and He, S. Y. 2013. Pseudomonas syringae pv. tomato DC3000: A model pathogen for probing disease susceptibility and hormone signaling in plants. Annu. Rev. Phytopathol. 51:473-498.

Yafei, C., Yong, Z., Xiaoming, Z., Peng, G., Hailong, A., Yuguang, D., Yingrong, H., Hui, L., and Yuhong, Z. 2009. Functions of oligochitosan induced protein kinase in tobacco mosaic virus resistance and pathogenesis related proteins in tobacco. Plant Physiol. Biochem. 47: 724-731.

Yang, A., Yu, L., Chen, Z., Zhang, S., Shi, J., Zhao, X., Yang, Y., Hu, D., and Song, B. 2017. Label-free quantitative proteomic analysis of chitosan oligosaccharide-treated rice infected with Southern rice black-streaked dwarf virus. Viruses 9:115.

Yin, H., Bai, X. F., and Du, Y. G. 2008. The primary study of oligochitosan inducing resistance to Sclerotinia sclerotiorum on Brassica napus. J. Biotechnol. 136:600-601.

Yin, H., Du, Y., and Dong, Z. 2016. Chitin oligosaccharide and chitosan oligosaccharide: Two similar but different plant elicitors. Front. Plant Sci. 7:522.

Yin, H., Li, S., Zhao, X., Du, Y., and Ma, X. 2006. cDNA microarray analysis of gene expression in Brassica napus treated with oligochitosan elicitor. Plant Physiol. Biochem. 44:910-916.

Yin, H., Li, Y., Zhang, H. Y., Wang, W. X., Lu, H., Grevsen, K., Zhao, X. M., and Du, Y. G. 2013. Chitosan oligosaccharides-triggered innate immunity contributes to oilseed rape resistance against Sclerotinia Sclerotiorum. Int. J. Plant Sci. 174:722-732.

Yin, H., Zhao, X. M., and Du, Y. G. 2010. Oligochitosan: A plant diseases vaccine-A review. Carbohydr. Polym. 82:1-8.

Zhang, H. Y., Wang, W. X., Yin, H., Zhao, X. M., and Du, Y. G. 2012. Oligochitosan induces programmed cell death in tobacco suspension cells. Carbohydr. Polym. 87:2270-2278.

Zhang, P. Y., and Chen, K. S. 2009. Age-dependent variations of volatile emissions and inhibitory activity toward Botrytis cinerea and Fusarium oxysporum in tomato leaves treated with chitosan oligosaccharide. J. Plant Biol. 52:332-339.

Zhang, S., Li, X., Sun, Z., Shao, S., Hu, L., Ye, M., Zhou, Y., Xia, X., Yu, J., and Shi, K. 2015. Antagonism between phytohormone signalling underlies the variation in disease susceptibility of tomato plants under elevated CO2. J. Exp. Bot. 66:1951-1963.

Zhang, W., Corwin, J. A., Copeland, D., Feusier, J., Eshbaugh, R., Chen, F., Atwell, S., and Kliebenstein, D. J. 2017. Plastic transcriptomes stabilize immunity to pathogen diversity: The jasmonic acid and salicylic acid networks within the Arabidopsis/Botrytis pathosystem. Plant Cell 29: 2727-2752

Zhao, X. M., She, X. P., Du, Y. G., and Liang, X. M. 2007. Induction of antiviral resistance and stimulary effect by oligochitosan in tobacco. Pestic. Biochem. Physiol. 87:78-84.

Zhao, Y., Thilmony, R., Bender, C. L., Schaller, A., He, S. Y., and Howe, G. A. 2003. Virulence systems of Pseudomonas syringae pv. tomato promote bacterial speck disease in tomato by targeting the jasmonate signaling pathway. Plant J. 36:485-499. 